\title{
DNA methylation involved in proline accumulation in response to osmotic stress in rice (Oryza sativa)
}

\author{
C.Y. Zhang ${ }^{1,2}$, N.N. Wang ${ }^{3}$, Y.H. Zhang, ${ }^{1}$ Q.Z. Feng ${ }^{1}$, C.W. Yang ${ }^{1}$ and B. Liu ${ }^{1}$ \\ ${ }^{1}$ Key Laboratory of Molecular Epigenetics of the Ministry of Education, \\ Northeast Normal University, Changchun, China \\ ${ }^{2}$ School of Food Production Technology and Biotechnology, \\ Changchun Vocational Institute of Technology, Changchun, China \\ ${ }^{3}$ Department of Agronomy, Jilin Agricultural University, Changchun, China \\ Corresponding authors: C.W. Yang / B. Liu \\ E-mail: chunwuyang@gmail.com / baoliu@nenu.edu.cn
}

Genet. Mol. Res. 12 (2): 1269-1277 (2013)

Received August 13, 2012

Accepted January 27, 2013

Published April 17, 2013

DOI http://dx.doi.org/10.4238/2013.April.17.5

\begin{abstract}
Proline accumulation is involved in plant osmotic stress tolerance. Given that DNA methylation is related to almost all metabolic processes through regulation of gene expression, we suspected that this epigenetic modification and proline biosynthesis are probably related. To test this, we investigated whether osmotic stress-induced proline accumulation is associated with DNA methylation modifications in rice. We assessed DNA methylation and expression of 3 key genes $(P 5 C R, P 5 C S$, and $\delta$-OAT) involved in proline biosynthesis, and measured proline content under both osmotic stress $(15 \%$ polyethylene glycol) and control conditions. After osmotic stress, selfed progenies of osmotic-stressed plants accumulated higher concentrations of proline in leaves under both normal conditions and under osmotic stress than the unstressed control plants. Concomitantly, under osmotic stress, the selfed progeny plants showed higher expression levels of $P 5 C S$ and $\delta$-OAT than the control. This up-regulated expression was stably inherited by the subsequent generation. Methylation-sensitive
\end{abstract}


Southern blotting indicated that 2 of the 3 genes, P5CS and $\delta$-OAT, had greater DNA demethylation in the selfed progenies than in the control. Apparently DNA demethylation facilitated proline accumulation by up-regulating expression of the $P 5 C S$ and $\delta$-OAT genes in response to osmotic stress.

Key words: Rice; Proline; Osmotic stress; DNA methylation

\section{INTRODUCTION}

Drought is one of the most prevalent abiotic stresses that limit crop productivity in many regions of the world. Plant response to drought is a very complex network affecting almost all processes in plant metabolism and development, including water balance, nutrient uptake and metabolism, and photosynthetic assimilation. Plant survival and growth under drought conditions result via adaptive processes involving altered ion uptake, stomatal regulation, and the accumulation of osmotic solutes such as proline. Proline accumulation is one of the most frequently reported physiological responses induced by drought and salt stress in plants and is believed to be involved in stress tolerance, although its precise role remains unclear (Lutts et al., 1999).

Proline accumulation during plant responses to osmotic stress is mainly due to increased synthesis and reduced degradation. In higher plants, proline is synthesized via 2 main pathways, the glutamate and ornithine pathways (Delauney and Verma, 1993; Roosens et al., 1998; Nanjo et al., 2003; Verbruggen and Hermans, 2008). Proline is synthesized via the glutamate pathway via 2 key enzymes, $\Delta^{1}$-pyrroline-5-carboxylate synthetase (P5CS, EC 1.5.1.12) and $\Delta^{1}$-pyrroline-5-carboxylate reductase (P5CR, EC 1.5.1.2) (Hu et al., 1992; Verbruggen and Hermans, 2008), whereas ornithine- $\delta$-aminotransferase ( $\delta$-OAT, EC 2.6.1.13) is a key enzyme of the ornithine biosynthesis pathway. In the glutamate pathway, reciprocal regulation of P5CS and P5C dehydrogenase (EC 1.5.1.12), a degradation enzyme, plays a key role in the control of proline levels (Kiyosue et al., 1996). Enzymolysis of synthesized proline from both pathways is mediated by proline dehydrogenase (EC 1.5.99.8) (Peng et al., 1996; Verbruggen et al., 1996). Although plant biologists have studied the proline biosynthesis pathway extensively, many questions remain. For example, how do plants regulate proline biosynthesis during growth and development? What are the tuning trajectories for its rapid synthesis in response to abiotic stresses?

Cytosine DNA methylation plays important roles in multiple fundamental cellular activities, including control of gene expression, maintenance of genomic integrity, formation and perpetuation of chromatin structure, and control of genomic imprinting (Rangwala and Richards, 2004; Tariq and Paszkowski, 2004; Chan et al., 2005). Studies in recent years have revealed that DNA methylation is labile and sometimes responsive to both internal and external perturbations and that biotic and abiotic stresses may cause heritable alterations in cytosine methylation patterns, which presumably represent adaptive responses, in both plants and animals (Richards, 2006). Several studies have shown that heritable phenotypic novelties may be related to heritable alterations in DNA methylation (Molinier et al., 2006; Boyko et al., 2007; Chinnusamy and Zhu, 2009). Given that DNA methylation is involved in almost all metabolic processes because it regulates gene expression, we suspected that epigenetic modification and proline biosynthesis are probably intrinsically interlaced. To test whether 
osmotic stress-induced proline accumulation is associated with DNA methylation modification, we investigated the transgenerational effects of osmotic stress on DNA methylation, the expression of key genes involved in proline metabolism, and proline accumulation in rice.

\section{MATERIAL AND METHODS}

\section{Plant materials}

'Matsumae', which used to be a major rice (Oryza sativa) cultivar in northeast China, was chosen as the test organism. Seeds were germinated and grown on Petri dishes for 6 days in a growth cabinet $\left(30^{\circ} \mathrm{C}\right.$ during the day and $25^{\circ} \mathrm{C}$ during the night, $16-/ 8$-h photoperiod at $\left.250 \mu \mathrm{mol} \cdot \mathrm{m}^{-2} \cdot \mathrm{s}^{-1}\right)$. Seedlings were transferred to buckets containing $2000 \mathrm{~mL}$ sterile nutrient solution for culture. The solution was replaced daily. The solution used in this study contained the components described by the International Rice Research Institute, including $1.44 \mathrm{mM}$ $\mathrm{NH}_{4} \mathrm{NO}_{3}, 0.32 \mathrm{mM} \mathrm{NaH} \mathrm{PO}_{4}, 0.6 \mathrm{mM} \mathrm{K}_{2} \mathrm{SO}_{4}, 1.0 \mathrm{mM} \mathrm{CaCl}_{2}, 1.6 \mathrm{mM} \mathrm{MgSO}_{4}, 0.072 \mathrm{mM}$ Fe-ethylenediaminetetraacetic acid, $0.2 \mathrm{mM} \mathrm{Na}_{2} \mathrm{SiO}_{3}, 9.1 \mu \mathrm{M} \mathrm{MnCl}_{2}, 0.154 \mu \mathrm{M} \mathrm{ZnSO}_{4}, 0.156$ $\mu \mathrm{M} \mathrm{CuSO}_{4}, 18.5 \mu \mathrm{M} \mathrm{H}_{3} \mathrm{BO}_{3}$, and $0.526 \mu \mathrm{M} \mathrm{H}_{2} \mathrm{MoO}_{4}, \mathrm{pH} 5.2$.

\section{Stress treatment}

After 14 days of growth in hydroponic medium, rice plants were subjected to osmotic stress by transferring them to buckets containing $2000-\mathrm{mL}$ treatment solution amended with the above nutrients and $15 \%$ polyethylene glycol for 4 days. We selected an osmotically stressed plant that showed the most obvious alteration in DNA methylation and its 16 selfed progenies (S1) to test the methylation state of P5CR, P5CS, and $\delta$-OAT using methylation-sensitive Southern blotting. We also tested the methylation state of an $O$. sativa retrotransposon 42 and the housekeeping gene Elongator, which are unrelated to osmotic stress. We compared proline content and the expression levels of P5CR, P5CS, and $\delta$-OAT in S1 plants and $3 \mathrm{~S} 2$ plants of this individual plant. Proline was measured using the ninhydrin method.

\section{DNA isolation and digestion}

Genomic DNA was isolated from expanded leaves using a cetyltrimethylammonium bromide method and purified with phenol extractions. The DNA was digested with the methylation-sensitive isoschizomer $M s p$ I. To ensure complete digestion, we used an excess of enzymes (10 U enzyme per $\mu \mathrm{g}$ DNA) and extended the incubation time to approximately 48 h. Restriction enzymes were purchased from New England Biolabs, Inc. (Beverly, MA, USA). Digested DNA was used for Southern blotting and inter-simple sequence repeat analysis.

\section{Methylation-sensitive amplification polymorphism (MSAP) analysis}

The MSAP method was carried out as reported by Dong et al. (2006). Rice genomic DNA was digested with an $M s p I$. In total, 1 pair of pre-selective primers and 19 pairs of selective primers were used for the amplifications (Table S1). The MSAP products were resolved using 5\% denaturing polyacrylamide gel electrophoresis and visualized with silver staining. 
Only clear and completely reproducible bands in 2 experiments using independent DNA extractions were scored.

\section{Methylation-sensitive Southern blotting}

Rice genomic DNA was digested with the MspI. The digested DNA was analyzed on $1 \%$ agarose gels and transferred to Hybond $\mathrm{N}^{+}$nylon membranes (Amersham Pharmacia Biotech, Piscataway, NJ, USA) using the alkaline transfer recommended by the supplier. These probes were prepared via sequence-specific polymerase chain reaction (PCR) amplifications using specific primers (Table S2). The authenticity of all PCR-amplified probe fragments was verified through sequencing. The fragments were gel purified and labeled with fluorescein11-2'-deoxyuridine 5'-triphosphate with the Gene Images Random Prime Labeling Module (Amersham Pharmacia Biotech). A hybridization signal was detected with the Gene Images CDP-Star detection module (Amersham Pharmacia Biotech) after washing at a stringency of $0.2 \mathrm{X}$ saline sodium citrate and $0.1 \%$ sodium dodecyl sulfate, twice for $50 \mathrm{~min}$. The filters were exposed to X-ray films.

\section{Quantitative real-time PCR analysis}

We extracted total RNA from the leaves of seedlings grown under stress and nonstress conditions using TRIzol reagent (Invitrogen). The RNA was treated with DNase I (Invitrogen), reverse-transcribed using SuperScript ${ }^{\mathrm{TM}}$ RNase H-Reverse Transcriptase (Invitrogen), and then subjected to real-time PCR analysis using gene-specific primers. The gene-specific primers are listed in Table S3. PCR amplification was conducted with an initial step at $95^{\circ} \mathrm{C}$ for $1 \mathrm{~min}$ followed by 45 cycles of $5 \mathrm{~s}$ at $95^{\circ} \mathrm{C}, 10 \mathrm{~s}$ at $60^{\circ} \mathrm{C}$, and $30 \mathrm{~s}$ at $72^{\circ} \mathrm{C}$. Amplification of the target gene was monitored using SYBR Green in every cycle. Amplifications of the rice Ubiquitin 5, Eukaryotic elongation factor-1 $\alpha$, and Atin 2 messenger RNA were used as an internal quantitative control (Jain et al., 2006; Zang et al., 2010). The relative expression of the target genes was calculated using the $\Delta \mathrm{Ct}$ method (Livak and Schmittgen, 2001). We optimized the PCR system, after which the amplification efficiencies of each target and reference gene were approximately equal.

\section{Statistical analysis}

Statistical analysis of the data was performed using the SPSS 13.0 statistical program (SPSS, Chicago, IL, USA). Measurements of proline content and gene expression were represented by an average of 3 replications and their standard errors, and each replicate consisted of a pool of 5 plants.

\section{RESULTS AND DISCUSSION}

\section{Alteration in DNA methylation induced by osmotic stress}

MSAP is a modified version of the standard amplified fragment length polymorphism technique that substitutes the original MseI enzyme with the HpaII or MspI methylation-sen- 
sitive enzymes. Using 19 pairs of selective EcoRI $+M s p$ I primer combinations (Table S1), we amplified 900 clear bands across the set of plant samples (including control plants and 14 individual osmotic stressed plants; Figure 1). By tabulating the number of bands representing the various MSAP patterns, we calculated the hypermethylation, hypomethylation, and total methylation levels (see Figure 1). Fourteen individual osmotically stressed plants showed small differences in total methylation levels but large difference in hypermethylation and hypomethylation pattern changes (see Figure 1). Compared with the non-stressed control plants, the osmotic stressed rice plants showed clearly altered DNA methylation patterns (see Figure 1).
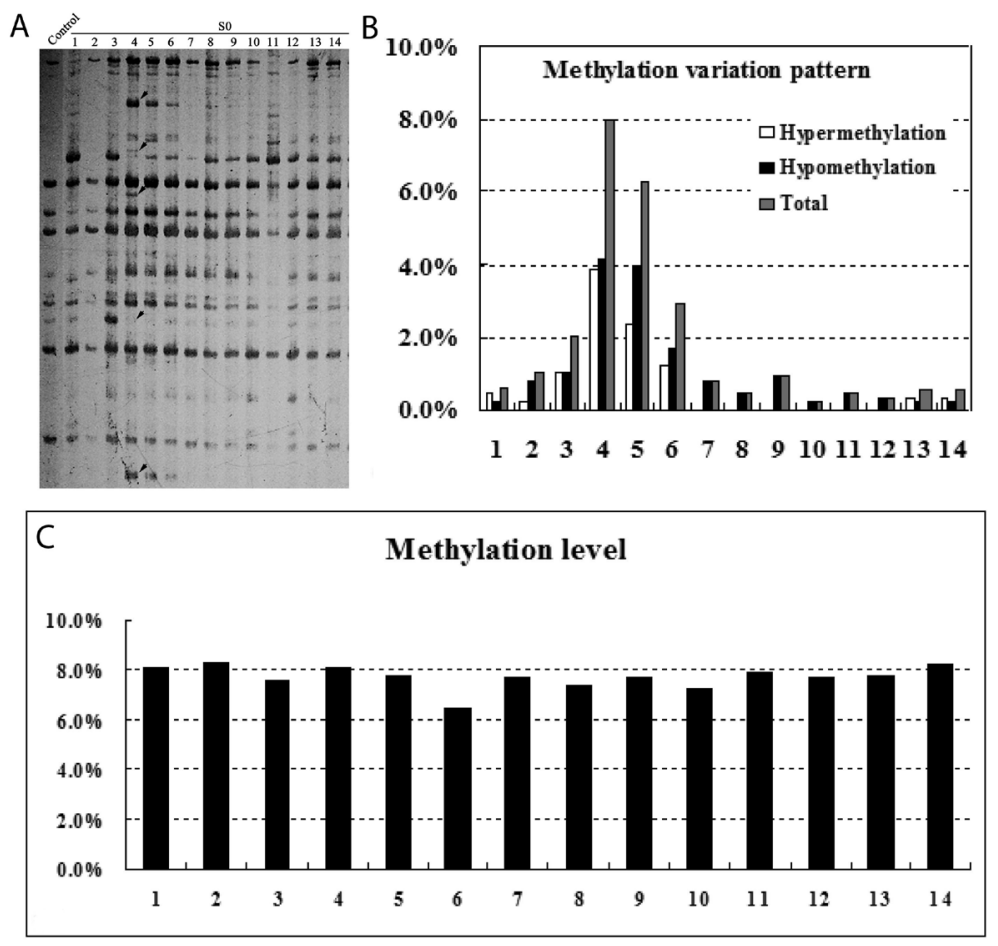

Figure 1. Effect of osmotic stress on DNA methylation state of rice plants revealed by MSAP analysis. A. Examples of altered DNA methylation patterns induced by osmotic stress; the methylation change was denoted by arrowheads. Lanes 1 to $14=$ osmotically stressed plants; Control = unstressed plant; S0 = individual osmotic stressed rice plant. The DNA was digested by a methylation-sensitive isoschizomer, Ms $p$ I. The digested DNA was analyzed by MSAP. B. DNA methylation alteration patterns of osmotic stressed plants. C. DNA methylation levels of osmotic stressed plants.

\section{Possible role of DNA methylation in proline accumulation}

We used methylation-sensitive Southern blotting analysis to investigate the role of DNA methylation in proline accumulation in response to osmotic stress. We selected an osmotically stressed plant that showed the most obvious alteration in DNA methylation patterns and its $16 \mathrm{~S} 1$ to test the methylation states of 3 genes, P5CR, P5CS, and $\delta$-OAT, known to be involved in osmotic stress tolerance (Figure 2) (Verbruggen and Hermans, 2008). We 
also compared expression levels of the 3 genes and proline content in an S1 plant and its 3 S2 plants. The Southern blotting results showed that under osmotic stress, 2 of the 3 tested genes, $P 5 C S$ and $\delta$-OAT, showed DNA demethylation in several stressed S1 plants but not in the immediately stressed plants (see Figure 2). By contrast, osmotic stress did not affect the methylation state of the third gene (P5CR), an $O$. sativa retrotransposon 42 and a housekeeping gene (Elongator; see Figure 2). This result suggested that the osmotic stress-induced DNA methylation changes are nonrandom.

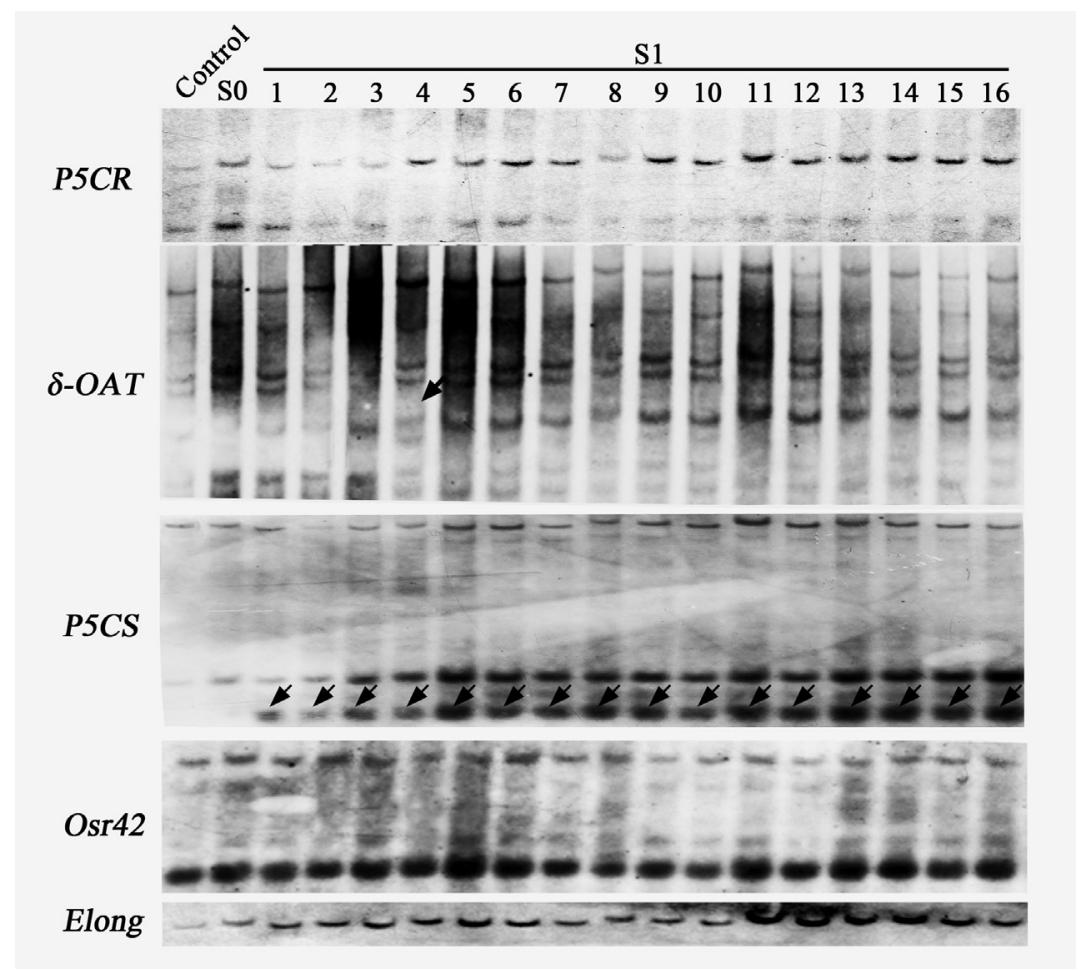

Figure 2. Transgenerational inheritance of altered DNA methylation patterns induced by osmotic stress to selfed progenies (S1) of the immediately stressed plants, revealed by methylation-sensitive Southern blotting. Control= unstressed plant; $\mathrm{S} 0=$ individual osmotic stressed rice plant; $\mathrm{S} 1=$ selfed progenies (lanes 1 to 16 ) of the stressed plant (S0). The rice plants were treated by $15 \%$ polyethylene glycol for 4 days. We selected an osmotic stressed plant that showed the most obvious alteration in DNA methylation and its $16 \mathrm{~S} 1$ plants to test methylation state of $P 5 C R, P 5 C S$, and $\delta$-OAT genes by methylation-sensitive Southern-blotting analysis. We also tested methylation state of a retrotransposon Osr42 and a housekeeping gene Elongator (Elong), which are known as unrelated to osmotic stress. P5CR $=\Delta^{1}$-pyrroline-5-carboxylase reductase; P5CS $=\Delta^{1}$-pyrroline-5-carboxylate synthetase; $\delta$-OAT $=$ ornithine- $\delta$-aminotransferase.

$P 5 C R, P 5 C S$ (glutamic acid pathway), and $\delta$-OAT (ornithine acid pathway) are 3 key genes of proline synthesis. Many studies have demonstrated that osmotic stress induces increases in $\delta$-OAT and P5CS abundance and activity (Roosens et al., 1998; Lutts et al., 1999). Thus, up-regulation of P5CS and $\delta$-OAT expression may contribute to the accumulation of proline in response to osmotic and salinity stress (Verslues and Sharma, 2010). We observed that in the immediately stressed plant generation, P5CS expression in rice leaves was not al- 
tered by osmotic stress. However, in selfed progeny of an osmotically stressed plant (S1) that showed DNA methylation changes, P5CS expression was also concomitantly up-regulated (see Figure 1). Interestingly, this altered gene expression pattern was also stably inherited in the S2 generation (Figure 3). Under osmotic stress, another proline synthesis key gene, $\delta$-OAT, was expressed in S1 and 2 S2 plants at levels much higher than those in the immediately stressed plant generation (control). The osmotic stress response of P5CR was similar to that of $\delta$-OAT.
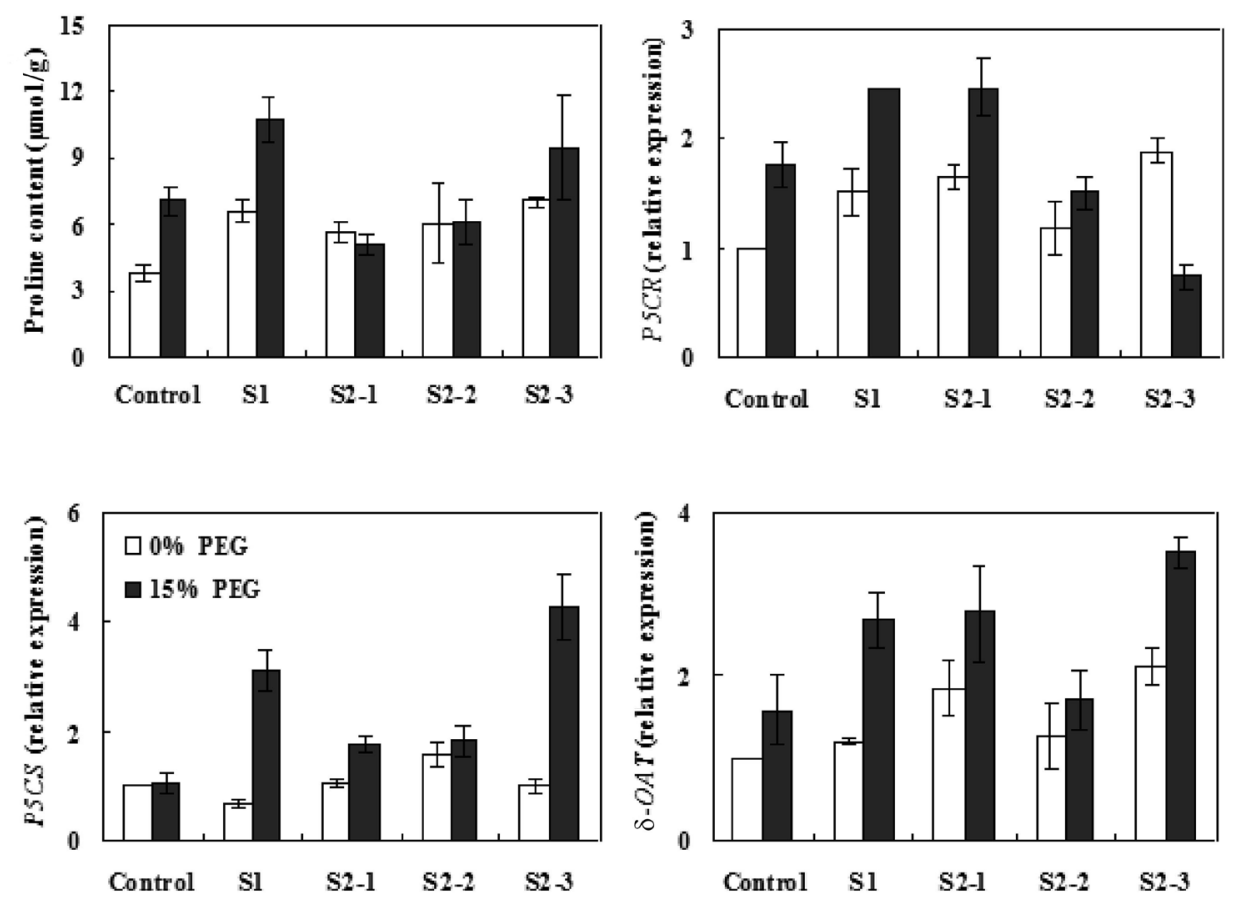

Figure 3. Effects of osmotic stress on proline content and expression of P5CR, P5CS, and $\delta$-OAT genes in rice leaves. Control $=$ normal rice plants grown under the same condition except the osmotic stress (Mock): $\mathrm{S} 1=$ selfed progeny of an osmotic stressed plant that showed most obvious alteration in DNA methylation; S2-1, S2-2, and $\mathrm{S} 2-3=3$ selfed progenies of this $\mathrm{S} 1$ plant. All rice plants were treated by $15 \%$ polyethylene glycol (PEG) for 4 days. $P 5 C R=\Delta^{1}$-pyrroline-5-carboxylase reductase; $P 5 C S=\Delta^{1}$-pyrroline-5-carboxylate synthetase; $\delta$-OAT $=$ ornithine$\delta$-aminotransferase.

In accordance with these changes in DNA methylation and gene expression, after the osmotic stress, the leaves of S1 and S2 plants accumulated concentrations of proline that were higher than those of the immediately stressed plant generation (control). In addition, under non-stress conditions, the S1 and S2 plants showed much higher concentrations of proline and higher expression levels of P5CS and $\delta$-OAT compared with those in the control plants (P $<0.5)$. These results indicated that the heritable up-regulation of P5CS and $\delta$-OAT may have contributed to the increased accumulation of proline in S1 and S2 plants even under non-stress conditions (see Figures 2 and 3). Namely, osmotic stress quickly generated a heritable phenotype within 1-2 generations that showed higher constitutive expression of relevant genes and 
proline accumulation. Epigenetic modification in the form of altered DNA methylation may have played a role in acquiring this heritable phenotype. Indeed, we found that under osmotic stress, both P5CS and $\delta$-OAT showed DNA demethylation in S1 plants but not in the immediately stressed plant generation, suggesting that DNA demethylation regulated expression of the 2 genes and resulted in their up-regulation.

The modulation in DNA methylation induced by abiotic stress may play a functional role in plant stress tolerance (Richards, 2006; Chinnusamy and Zhu, 2009; Karan et al., 2012). Biotic and abiotic stresses may cause alterations in DNA methylation patterns in both plants and animals (Richards, 2006). Most of these stress-induced epigenetic modifications are reset to the basal level once the stress is relieved, but some of the modifications may be stable - that is, may be carried forward as "stress memory" or organismal progenies (Chinnusamy and Zhu, 2009). Thus, alterations in methylation patterns could be considered part of an evolutionarily meaningful plant protection mechanism in response to environmental stress.

Proline, as a compatible osmolyte, is widely believed to accumulate rapidly in response to water deficiency or osmotic stress (Saradhi et al., 1995; Hare and Cress, 1997; Siripornadulsil et al., 2002; Verbruggen and Hermans, 2008; Mattioli et al., 2009). However, the function of proline in drought or osmotic tolerance is still under debate by plant biologists (Siripornadulsil et al., 2002; Verbruggen and Hermans, 2008). Recent findings have suggested that proline may also play a role in flowering and development both as a metabolite and a signal molecule (Mattioli et al., 2009). In any case, proline clearly plays crucial roles not only in drought tolerance but also in tolerance to other stresses such as salinity, high $\mathrm{pH}$, and cold. Our results suggested that changes in DNA methylation (demethylation) as a result of osmotic stress facilitate proline accumulation via the up-regulation of proline metabolism-related gene expression (e.g., P5CS and $\delta$-OAT), which is likely an adaptive response to the stress.

\section{ACKNOWLEDGMENTS}

Research supported by the Project of the Jilin Provincial Science \& Technology Department (\#201205048), the National Natural Science Foundation of China (\#30970232 and \#30870178), and the Fundamental Research Funds for the Central Universities (\#11QNJJ020).

\section{Supplementary material}

\section{REFERENCES}

Boyko A, Kathiria P, Zemp FJ, Yao Y, et al. (2007). Transgenerational changes in the genome stability and methylation in pathogen-infected plants: (virus-induced plant genome instability). Nucleic Acids Res. 35: 1714-1725.

Chan SW, Henderson IR and Jacobsen SE (2005). Gardening the genome: DNA methylation in Arabidopsis thaliana. Nat. Rev. Genet. 6: 351-360.

Chinnusamy V and Zhu JK (2009). Epigenetic regulation of stress responses in plants. Curr. Opin. Plant Biol. 12: 133-139. Delauney AJ and Verma DPS (1993). Proline biosynthesis and osmoregulation in plants. Plant J. 4: 215-223.

Dong ZY, Wang YM, Zhang ZJ, Shen Y, et al. (2006). Extent and pattern of DNA methylation alteration in rice lines derived from introgressive hybridization of rice and Zizania latifolia Griseb. Theor. Appl. Genet. 113: 196-205.

Hare P and Cress W (1997). Metabolic implications of stress-induced proline accumulation in plants. Plant Growth Regul. 21: 79-102.

Hu CA, Delauney AJ and Verma DP (1992). A bifunctional enzyme (delta 1-pyrroline-5-carboxylate synthetase) catalyzes the first two steps in proline biosynthesis in plants. Proc. Natl. Acad. Sci. U. S. A. 89: 9354-9358. 
Jain M, Nijhawan A, Tyagi AK and Khurana JP (2006). Validation of housekeeping genes as internal control for studying gene expression in rice by quantitative real-time PCR. Biochem. Biophys. Res. Commun. 345: 646-651.

Karan R, DeLeon T, Biradar H and Subudhi PK (2012). Salt stress induced variation in DNA methylation pattern and its influence on gene expression in contrasting rice genotypes. PLoS One 7: e40203.

Kiyosue T, Yoshiba Y, Yamaguchi-Shinozaki K and Shinozaki K (1996). A nuclear gene encoding mitochondrial proline dehydrogenase, an enzyme involved in proline metabolism, is upregulated by proline but downregulated by dehydration in Arabidopsis. Plant Cell 8: 1323-1335.

Livak KJ and Schmittgen TD (2001). Analysis of relative gene expression data using real-time quantitative PCR and the $2^{-\Delta \Delta C \mathrm{~T}}$ method. Methods 25: 402-408

Lutts S, Majerus V and Kinet JM (1999). NaCl effects on proline metabolism in rice (Oryza sativa) seedlings. Physiol. Plant. 105: 450-458.

Mattioli R, Costantino P and Trovato M (2009). Proline accumulation in plants: not only stress. Plant Signal. Behav. 4: 1016-1018.

Molinier J, Ries G, Zipfel C and Hohn B (2006). Transgeneration memory of stress in plants. Nature 442: 1046-1049.

Nanjo T, Fujita M, Seki M, Kato T, et al. (2003). Toxicity of free proline revealed in an Arabidopsis T-DNA-tagged mutant deficient in proline dehydrogenase. Plant Cell Physiol. 44: 541-548.

Peng Z, Lu Q and Verma DP (1996). Reciprocal regulation of delta 1-pyrroline-5-carboxylate synthetase and proline dehydrogenase genes controls proline levels during and after osmotic stress in plants. Mol. Gen. Genet. 253: 334-341.

Rangwala SH and Richards EJ (2004). The value-added genome: building and maintaining genomic cytosine methylation landscapes. Curr. Opin. Genet. Dev. 14: 686-691.

Richards EJ (2006). Inherited epigenetic variation - revisiting soft inheritance. Nat. Rev. Genet. 7: 395-401.

Roosens NH, Thu TT, Iskandar HM and Jacobs M (1998). Isolation of the ornithine-delta-aminotransferase cDNA and effect of salt stress on its expression in Arabidopsis thaliana. Plant Physiol. 117: 263-271.

Saradhi PP, Alia, Arora S and Prasad KV (1995). Proline accumulates in plants exposed to UV radiation and protects them against UV induced peroxidation. Biochem. Biophys. Res. Commun. 209: 1-5.

Siripornadulsil S, Traina S, Verma DP and Sayre RT (2002). Molecular mechanisms of proline-mediated tolerance to toxic heavy metals in transgenic microalgae. Plant Cell 14: 2837-2847.

Tariq M and Paszkowski J (2004). DNA and histone methylation in plants. Trends Genet. 20: 244-251.

Verbruggen $\mathrm{N}$ and Hermans C (2008). Proline accumulation in plants: a review. Amino Acids 35: 753-759.

Verbruggen N, Hua XJ, May M and Van Montagu M (1996). Environmental and developmental signals modulate proline homeostasis: evidence for a negative transcriptional regulator. Proc. Natl. Acad. Sci. U. S. A. 93: 8787-8791.

Verslues PE and Sharma S (2010). Proline metabolism and its implications for plant-environment interaction. Arabidopsis Book 8: e0140.

Zang A, Xu X, Neill S and Cai W (2010). Overexpression of OsRAN2 in rice and Arabidopsis renders transgenic plants hypersensitive to salinity and osmotic stress. J. Exp. Bot. 61: 777-789. 